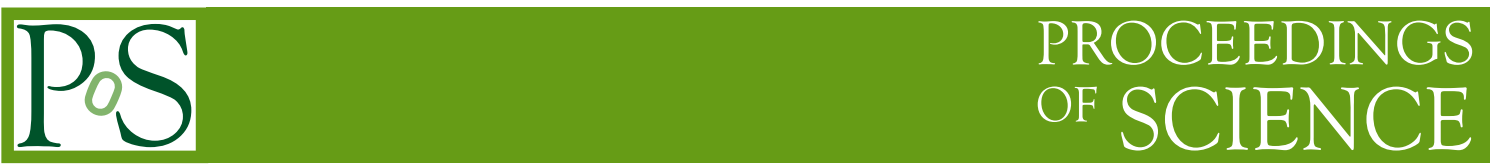

\title{
Details of T2K Oscillation Analysis
}

\author{
C. Bronner*t \\ Kamioka Observatory, ICRR, The University of Tokyo \\ E-mail: christophe.bronnereipmu.jp
}

\begin{abstract}
Neutrino oscillations are studied in T2K by comparing the observed data at the far detector to the predictions for different hypotheses to make statistical inferences. The predictions are made using a model of the experiment, constructed from a set of models and simulations tuned to data to predict the neutrino flux reaching the detectors, and describe neutrino interactions as well as the detectors response. Using data corresponding to an exposure of $1.49 \times 10^{21}$ proton on targets (POT) in neutrino mode, and $1.63 \times 10^{21}$ POT in anti-neutrino mode, T2K excluded conservation of CP symmetry in neutrino oscillation at the $2 \sigma$ level. An excess of observed events compared to the predictions in one of the samples has a strong contribution to this result, and it was found that the p-value to observe such an excess in 1 of the 5 analysis samples was between $5.8 \%$ and $11.3 \%$ depending on the values of the oscillation parameters assumed.
\end{abstract}

The 21st international workshop on neutrinos from accelerators (NuFact2019)

August 26 - August 31, 2019

Daegu, Korea

\footnotetext{
*Speaker.

${ }^{\dagger}$ On behalf of the $\mathrm{T} 2 \mathrm{~K}$ collaboration
} 


\section{Introduction}

The Tokai to Kamioka (T2K) experiment [1] is a long baseline neutrino oscillation experiment located in Japan. A beam of neutrinos is produced using an accelerator at J-PARC, and travels 295 $\mathrm{km}$ before reaching the far detector Super-Kamiokande (Super-K) where the neutrinos are detected after oscillation. To produce the beam, accelerated protons are sent to collide on a graphite target, which produces hadrons including charged pions and kaons that will decay in flight into neutrinos. A set of magnetic horns allows to selectively focus hadrons based on their charge, resulting in a beam made predominantly of neutrinos or anti-neutrinos depending on the polarity of the horns. The obtained (anti-)neutrinos are mainly of the muon flavor, with a $1 \% v_{e}$ contamination at the flux peak energy. The experiment uses the off-axis technique: the far detector is not located on the axis of the beam, but in a direction making a $2.5^{\circ}$ angle with this axis. As a result, Super-K sees a narrow band beam, peaked at the maximum of oscillation probability. In this paper, we will describe the way T2K data are analyzed to study neutrino oscillations, and look more in detail at certain aspects of the results obtained using the first 9 years of data.

\section{T2K oscillation analysis}

In $\mathrm{T} 2 \mathrm{~K}$, neutrino oscillations are studied using the rate and spectra of the electron and muon neutrino candidate events detected at Super-K. The observations are compared to the predictions from the standard 3 flavor oscillation model for different values of the oscillation parameters to measure those parameters, or for different hypotheses (for example normal and inverted mass hierarchies) to test those hypotheses. The predictions are based on a simulation of the experiment tuned to data. The flux and interaction part of the model is tested in the near detectors, allowing us to tune the nominal predictions at the far detector and constrain their uncertainties.

The flux of neutrinos reaching the detectors is predicted using a series of simulations. The inputs to this simulation chain are primarily the properties of the proton beam measured by the beam monitors. The production of hadrons from interactions in the target and the propagation and decay of those hadrons in the secondary beamline are simulated to predict the neutrino flux, using data from the NA61/SHINE experiment [2] to tune the hadron production model. The resulting flux prediction has an uncertainty ranging between $8 \%$ and $12 \%$, depending on the neutrino flavor and energy. At the T2K beam energies, the dominant interaction mode is the charged-current quasielastic (CCQE) one, but other interaction modes will also contribute and populate the region of interest for the studies of neutrino oscillations, especially when the neutrino energy is not properly reconstructed. Models for each of the interaction modes are selected based on comparisons with external and near detector data, and the nominal predictions are obtained from the NEUT interaction generator [3], version 5.3.2. Systematic uncertainties on neutrino interactions are modelled through uncertainties on the model parameters for the most important modes, with additional shape and normalization uncertainties for certain modes.

The combination of the flux and interaction models is then tested in the near detectors, taking into account the systematic uncertainties of those detectors. For this, charged current (CC) $v_{\mu}$ interactions occurring in the tracker of the ND280 near detector are selected. The tracker is made of 2 fine grained detectors (FGD) acting as target mass (containing both carbon and water targets) 
and 3 time projection chambers providing precise reconstruction, and is located off-axis from the beam (in the Super-K direction) inside a magnet providing a $0.2 \mathrm{~T}$ field. The events are separated in samples enriched in different interaction modes based on the reconstructed event topologies, and corresponding to interaction on different target materials based on the FGD in which the interaction occurred. In anti-neutrino running mode, additional samples are used to constrain the wrong sign background ( $v$ component of the mainly $\bar{v}$ beam). Two different analysis codes, relying on different methods, are used to fit the model to the near detector data. The first code uses a classic gradient descent method to minimize the likelihood and obtain best fits and errors on the parameter values, while the second one uses a Markov Chain Monte Carlo (MCMC) method to obtain the posterior probability distributions of the parameters. The 2 codes give consistent results, giving confidence in the fact that the fits work properly and that the results are not too sensitive to the fitting method used. The result of this near detector analysis is a set of tuned nominal values and uncertainties for the parameters describing flux and interaction systematic uncertainties in the model, with their correlations. The tuned model gives predictions at the far detector with significantly reduced uncertainties on the rate and spectra compared to the initial model.

To be able to study oscillations at the far detector, we need to select events corresponding to CC interactions, separate them based on the flavor of the neutrino which interacted, and build observables correlated to the neutrino energy. Super-K is a water Cerenkov detector, and can only detect photons and charged particles that have high enough momentum. In practice at T2K energies, only photons, pions and charged leptons (excluding taus) are visible, which limits the ability to reconstruct the $v$ energy. However, since the direction of the incoming neutrino is known, it is possible to reconstruct its energy using only the momentum and direction of one of the produced particles for interactions where only 2 particles are produced. One such interaction is the CCQE interaction, in which only the charged lepton produced is visible in the detector. This lepton can then be used to separate between $v_{\mu} / \bar{v}_{\mu}$ and $v_{e} / \bar{v}_{e}$ CC interactions as Super-K has good ability to distinguish between $\mathrm{e}^{ \pm}$and $\mu^{ \pm}$. 4 CCQE enriched samples are built by selecting events where only one particle is visible as a ring in the detector, and separating them between $v$ and $\bar{v}$ running mode and whether the ring is electron-like (1Re samples) or muon-like (1R $\mu$ samples). A fifth sample is added, targeting CC resonant $\pi^{+}$production from $v_{e}$ by selecting events recorded in $v$-mode where one electron-like ring and one Michel electron were observed (CC1 $\pi^{+}$sample).

For the oscillation fits, three different analysis codes are used. They differ by the way they use the near detector data ( 2 of them use the results of the near detector analysis, while the last one does a simultaneous fit of near and far detector data), the fitting method used and the kinematic information about the electron-like events they use. Those 3 analysis were found to give identical results in sensitivity studies, and similar but slightly different results in the data fit. Finally, a procedure called simulated data studies is used to look for possible biases coming from neutrino interaction related uncertainties not taken into account by the systematic parameters. In this procedure, we compare the intervals obtained for the oscillation parameters when fitting our model to data generated with nominal and modified interaction models. We found that a mismodelling of the nucleon binding energy could create a significant bias on $\sin ^{2}\left(\theta_{23}\right)$ and $\Delta m_{32}^{2}$ measurements, and added an ad-hoc systematic parameter to cover this possible bias. A number of modified interaction models were found to change the intervals obtained for $\Delta m_{32}^{2}$, and the likelihood obtained in the fits will be smeared in the $\Delta m_{32}^{2}$ direction to take this into account. 


\section{Neutrino oscillation results using the run 1-9 data}

\subsection{Results}

The latest T2K neutrino oscillation results are obtained from the analysis of the data recorded at the far detector during the first 9 years of data taking of the experiment. This corresponds to an exposure of $1.49 \times 10^{21}$ proton on targets (POT) in $v$-mode, and $1.63 \times 10^{21}$ POT in $\bar{v}$-mode. Using the results of the reactor experiments to constrain the value of $\theta_{13}$ [4], we obtained a $2 \sigma$ exclusion of the conservation of CP symmetry: the $\Delta \chi^{2}$ values for $\delta_{C P}=0$ and $\delta_{C P}=\pi$ are larger than the $2 \sigma$ critical values obtained using the Feldman-Cousins method [5] as can be seen on the left plot of Fig. 1. We also find a preference for the octant $\sin ^{2} \theta_{23}>0.5$, although our results are compatible with maximum mixing and do not exclude the other octant at the $1 \sigma$ level. Finally, we find a preference for the normal mass hierarchy, with a posterior probability ranging between $87.7 \%$ and $91.1 \%$ depending on the far detector analysis used.
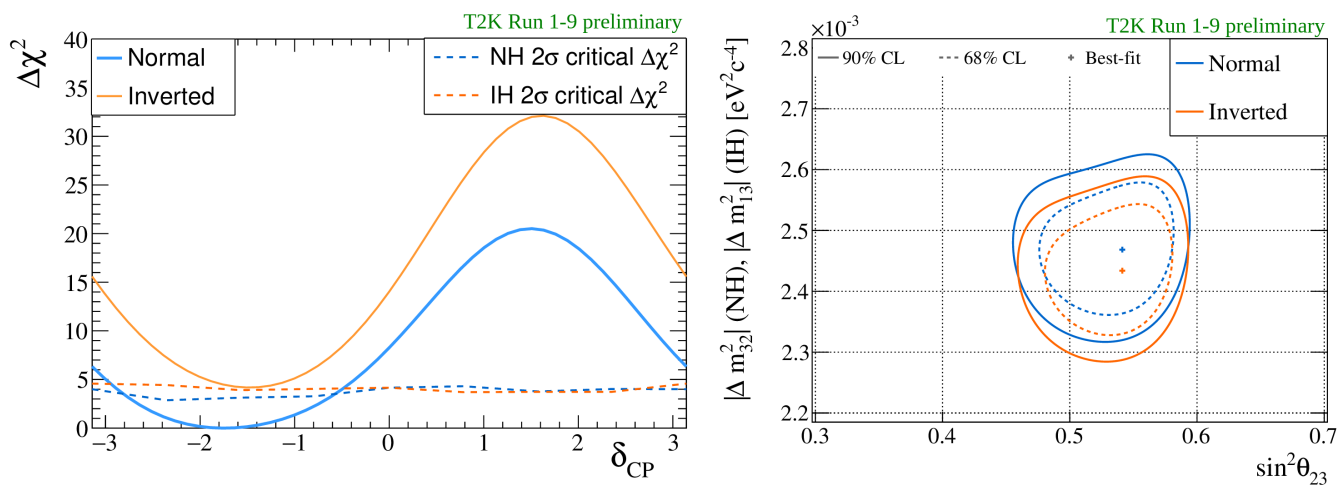

Figure 1: Measurement of the oscillation parameters using T2K run 1-9 data: $\Delta \chi^{2}$ as a function of $\delta_{C P}$ (left) and CL regions obtained with the fixed $\Delta \chi^{2}$ method for the atmospheric parameters (right).

\subsection{Data/MC difference in the number of events}

Table 1 shows the comparison between the number of events observed in each sample at the far detector and the predictions for $\sin ^{2} \theta_{23}=0.528, \Delta m_{32}^{2}=2.509 \times 10^{-3} \mathrm{eV}^{2} / \mathrm{c}^{4}, \sin ^{2} \theta_{13}=0.0219$ and $\delta_{C P}=-\pi / 2$. We find a good agreement for 3 of the 5 samples, a small deficit of events in the $v$-mode $1 \mathrm{R} \mu$ sample and an excess of events in the $v$-mode $\mathrm{CC} 1 \pi^{+}$sample. To evaluate the effects of those differences on the CP violation result, the fit of the run 1-9 data for $\delta_{C P}$ was redone replacing the observations by the MC predictions for one sample at the time (Fig. 2). We can see that in all cases, the obtained $\Delta \chi^{2}$ curve is above the sensitivity curve (in red, all samples are MC), and that the most visible changes for $\delta_{C P}=-\pi$ come from replacing the data by the predictions for the $v$-mode $1 \mathrm{R} \mu$ and $\mathrm{CC} 1 \pi^{+}$samples. In particular, in the case of the $\mathrm{CC} 1 \pi^{+}$sample the obtained $\Delta \chi^{2}$ for $\delta_{C P}=-\pi$ is below the $2 \sigma$ critical value, meaning that the $2 \sigma$ exclusion of $\delta_{C P}=-\pi$ does not occur without the high rate of $\mathrm{CC} 1 \pi^{+}$events. We therefore wanted to evaluate the likelihood of such an excess according to our model. We first confirmed that the kinematic distribution of the observed events for this sample matched the predictions, and then calculated the probability 
to observe 15 or more events in this sample according to our model. The obtained p-values were respectively of $2.49 \%$ and $1.34 \%$ for true values of the oscillation parameters corresponding to the $\mathrm{T} 2 \mathrm{~K}$ only and $\mathrm{T} 2 \mathrm{~K}+$ reactor best fits. Taking into account the trial factor, the probabilities to observe such an excess in at least one of the 5 samples were found to be $11.3 \%$ and $5.8 \%$ respectively.

\begin{tabular}{lcc}
\hline Sample & MC & Data \\
\hline$v$-mode $1 \operatorname{Re}$ & 74.46 & 75 \\
$v$-mode $1 \mathrm{R} \mu$ & 272.34 & 243 \\
$\bar{v}$-mode $1 \mathrm{Re}$ & 17.15 & 15 \\
$\bar{v}$-mode $1 \mathrm{R} \mu$ & 139.47 & 140 \\
$\nu$-mode $\mathrm{CC} 1 \pi^{+}$ & 7.02 & 15 \\
\hline
\end{tabular}

Table 1: Comparison between the number of events observed in each sample and the predictions.

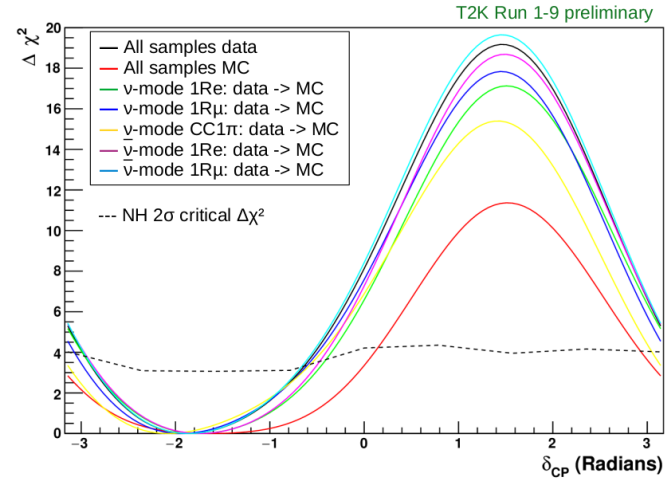

Figure 2: Change of the result of the fit of the T2K run 1-9 data for $\delta_{C P}$ when observations get replaced by predictions for the different samples.

\subsection{Mass hierarchy results}

$\mathrm{T} 2 \mathrm{~K}$ uses as primary result for the mass hierarchy $(\mathrm{MH})$ the posterior probabilities of the normal $(\mathrm{NH})$ and inverted $(\mathrm{IH})$ hierarchies, assuming equal prior probabilities. We have checked how the posterior probabilities obtained changed as a function of the prior probabilities assumed, and confirmed that our data contained information about the MH. The frequentist properties of the posterior probabilities were also checked, by comparing how often we rejected the true and false hypotheses when the criteria to reject a hypothesis was the other one having a posterior probability larger than $95 \%$. Finally, we computed frequentist results for the mass hierarchies by evaluating how often we expected to obtain a result more NH-like (resp. IH-like) than the data fit result if the true hierarchy was inverted (resp. normal). We obtained p-values of $4.87 \times 10^{-3}$ for the $\mathrm{IH}$ and $6.5 \times 10^{-2}$ for the $\mathrm{NH}$. Both p-values are low, and it would therefore be misleading to claim exclusion of one hierarchy based only on the p-value for this hypothesis.

\section{References}

[1] K. Abe et al. (T2K collaboration), Nucl. Instrum. Meth., A659,106 (2011)

[2] N. Abgrall et al., Nucl. Instrum. Meth., A701, 99 (2013)

[3] Y. Hayato, Acta Phys. Polon. B40 (2009) 2477-2489

[4] M. Tanabashi et al. (Particle Data Group), Phys. Rev. D98, 030001 (2018).

[5] G. J. Feldman and R. D. Cousins, Phys. Rev. D57, 3873(1998) 